\title{
Application of Genetic Neural Network in Power Battery Charging State-of-Charge Estimation
}

\author{
Yongqin Zhou \\ College of Electrical \& Electronic Engineering. Harbin University of Science \& Technology \\ Harbin, China \\ zyqemail@163.com \\ Chao Bai and Jinlei Sun \\ College of Electrical \& Electronic Engineering. Harbin University of Science \& Technology \\ Harbin, China \\ baichaoabcd@163.com,sj12001@163.com
}

\begin{abstract}
With global non-renewable resources and environmental issues becoming more apparent, the development of new energy vehicles have become the trend of auto industry. Hybrid vehicle becomes the key development of new energy vehicles with its long distance, low pollution, low fuel consumption characteristics and so on. The battery performances directly influence the quality of the whole vehicle performance. Considering the importance of the battery state of charge (SOC) estimation and the nonlinear relationship between the battery SOC and the external characteristic, genetic algorithm (GA) and back propagation (BP) neural network are proposed. Because of the strong global search capability of the genetic algorithm and the generalization ability of $B P$ neural network, the hybrid vehicle Ni-MH power battery GA-BP charging model is designed. In this approach, the network training speed is superior to the traditional BP network. According to the real-time data of the batteries, the optimal solution can be concluded in a short time and with high estimation precision.
\end{abstract}

Index Terms-BP neural network, genetic algorithm, power battery

\section{INTRODUCTION}

Vehicle power battery online State-of Charge (SOC) estimation has been recognized as a technical problem all over the world for a long time, it also attracted the attention of many scholars both at home and abroad. Factors such as battery temperature, the polarization, cycle magnification, the last charge and discharge status and battery life bring effect to the estimation. Thus, the battery SOC is a nonlinear system, People also carried out a series of algorithms, but the effect was not ideal, these methods all have defects. So the accuracy and reliability of the battery SOC estimation remains to be improved.

SOC is the internal characteristics of battery which

Heilongjiang provincial science and technology key project;

The development of hybrid car based on hafei HFJ7182.Projict Number: GB08A306 could not be measured, the only method is to measure the external characteristic of battery and estimate it with a reasonable algorithm. Comparing with the intelligent algorithm, the accuracy of traditional algorithm in calculation and real-time online has certain disparity. In the estimation of intelligent algorithm, BP neural network has been widely used, it has the basic characteristic of non-linear and parallel structure and study ability. There is no need to describe the precise relationship between input and output of the formula, as long as the reasonable selection of input parameters and output parameters are determined, input and output relationship are identified in the network training. But there are also some defects and deficiencies of its own, such as trapped in local minimum, slow convergence and the effects caused by shocks and so on. In view of the deficiencies mentioned above, if a method is used to optimize the initial value before the BP algorithm, using the optimized weights and threshold value as the initial value of the $\mathrm{BP}$ network can solve these problems.

In the running process of hybrid vehicle, the charging condition is essential, while the charging quality of the battery directly affect the battery life and continual mileage. Energy can be recovered when the vehicle is running, if the charging process is not controlled, it's very easy to damage the battery, so the use of motor controllers for achieving closed loop control to charge the batteries at constant current. For the SOC estimation under the condition of constant current, if the traditional Ah method is taken, the estimation will be affected by running time and testing accurate.

For constant current charging of the power battery SOC estimation problem, combining with the uncertain nonlinear relationship between battery SOC and the external characteristic parameters of batteries. This paper presents a battery SOC estimation of genetic algorithm back propagation (GA-BP) neural network which combined the genetic algorithm with $\mathrm{BP}$ algorithm. The combination can not only develop the mapping and generalization ability of neural network, but also let neural network have a fast convergence rate and strong learning ability. Comparative analysis of the simulation 
shows that this method is faster than the traditional BP neural network training and closer to the real SOC.

\section{Comon SOC Estimation Methods And Problems}

\section{A. Mathematical Model}

Mathematical model is essentially based on the internal physical and chemical reactions of battery, using thermodynamics and kinetics equations to analyze actual problems. A model for Ni-MH battery has been established by foreign scholars, including the one dimensional slab model, two-dimensional model, threedimensional model and compound model.

\section{B. Open-circuit Voltage Method}

Open-circuit voltage method is based on the corresponding relationship between open circuit voltage and the discharge depth of the battery, measuring the open circuit voltage to estimate the battery SOC. In practice, the Ni-MH battery is relatively stable in a long zone of discharge voltage, this method is difficult to estimate accurately. Furthermore the open circuit voltage during the charging process is also difficult to obtain.

\section{Ah Counting Method}

Ah counting method is the method most commonly used for estimating SOC. This method is simple, which just needs to collect current and time, by calculating the total energy to obtain SOC. This method is very sensitive to the accuracy of the current collection, while the collecting error of the current will be long-term accumulated, and the error will be much bigger in the case of high temperature and current volatility.

\section{Kalman Filter Recursive Algorithm}

In the SOC estimation based on Kalman filter [1], the battery is considered a dynamic system, while the SOC is an internal system state variable. The method needs to choose the equations of the dynamic system, recursive process also involves a complex matrix inversion. As for the Kalman filter recursive algorithm, the choice is very sensitive to initial values, the error lead to the initial estimated even worse, initial SOC0 can be set by the open circuit voltage, but the other initial value for the recurrence is difficult to determine.

\section{E. Neural Network Method}

Battery is a highly nonlinear system, its chargedischarge process is difficult to establish accurate mathematical model. Neural network has the basic characteristics of non-linear, with parallel structure and learning ability, while the external stimulus can give the corresponding output, so it can simulate the battery dynamics to estimate the SOC. Commonly used input variables are voltage, current, cumulative release of power, temperature, internal resistance and environmental temperature and so on. The selection of the neural network input variable and the number of the variables directly affect the accuracy and the computational. Neural network method is suitable for all kinds of batteries, the disadvantage is that it needs to train a large of number of reference data, estimation error is affected greatly by the training data and training methods.

\section{BATTERY SOC BASED ON GA-BP}

\section{A. Battery SOC Dedinition and Influencing Factors}

For the hybrid electric vehicle battery management system, the remaining capacity of the estimate is the core of the battery management system, an accurate estimate of remaining capacity is available for the drivers to estimate vehicle mileage and replenish energy. A widely used definition of the SOC is that the value is equal to the remaining battery capacity of the ratio of total battery capacity,

$$
S O C=\frac{Q_{c}}{Q_{\text {full }}}
$$

Where, $Q_{c}$ represents the remaining capacity of battery, $Q_{\text {full }}$ is the total capacity of battery, generally measured the battery capacity by Ah. For example: in this paper, the nominal capacity of $\mathrm{Ni}-\mathrm{MH}$ power battery is $8 \mathrm{Ah}$ that means 8A constant current discharge can last an hour. According to the definition above, the value of SOC is between 0 and 1 , but considering the influence of the over-charge and over-discharge to the battery life, it's needed to limit the battery SOC between 0.2 and 0.8 .

Battery charge and discharge mechanism is a complex electrochemical reaction, which determines that the battery's SOC can not be obtained by measurement, but by measuring the parameters of external characteristic [2].

The battery can be considered highly nonlinear system. In order to estimate more accurately, it is necessary to take the factors that affect the battery into account. The following TABLE I lists the reasons and factors.

\section{B. GA-BP Model}

The commonly used SOC estimation methods have disadvantages of the respective applications, and there is no way to really estimate accurately and reliably, so considering the actual problem of the power $\mathrm{Ni}-\mathrm{MH}$ battery SOC estimation, the model of genetic algorithms and BP neural network is established.

BP neural network has been widely used for its good performance, about $80 \%$ to $90 \%$ of the artificial neural network model is BP neural network, or its derivative forms [3]. And this network has advantages of simple structure and fast training speed. It has been documented in theory, without limiting the number of nodes in the hidden layer, only one hidden layer of BP neural network can realize any nonlinear mapping. Therefore, the three layers of BP network are selected, namely, input layer, hidden layer and output layer. BP neural network algorithm based primarily on the network input and output data set corresponding training the network to establish the corresponding network input and output mapping. Network includes signal forward propagation and the error back propagation. The samples pass from the input layer, through the hidden layer processing then transmit to the output layer during signal forward propagation. If the actual output of the output layer does 
TABLE I

INFLUENCE FACTOR AND MECHANISM

\begin{tabular}{|c|c|}
\hline $\begin{array}{l}\text { Influence } \\
\text { factor }\end{array}$ & Influence mechanism \\
\hline $\begin{array}{c}\text { Battery } \\
\text { temperature }\end{array}$ & $\begin{array}{l}\text { With the increasing of temperature, electrolyte } \\
\text { activity increases, particle motion increases, the } \\
\text { utilization of material increased, capacity increases. } \\
\text { Conversely, low temperature will lead to capacity } \\
\text { reduced. }\end{array}$ \\
\hline Battery aging & $\begin{array}{l}\text { Battery continuously charge and discharge its } \\
\text { internal structure and materials will occur aging } \\
\text { recession. In general, battery aging is affected by the } \\
\text { number of recycling, charging ways, discharging } \\
\text { current and discharging depth, and so on. }\end{array}$ \\
\hline $\begin{array}{l}\text { Battery } \\
\text { internal } \\
\text { resistance }\end{array}$ & $\begin{array}{l}\text { Battery internal resistance can be divided into ohm } \\
\text { resistance and polarization resistance. Polarization } \\
\text { resistance generated when charging and discharging. } \\
\text { The ohm resistance generates mainly by the } \\
\text { terminal, the electrolyte membrane and electrode } \\
\text { materials. Therefore, the resistance is related to the } \\
\text { conductivity of the electrolyte and battery structure, } \\
\text { its changes also have an impact on the capacity. }\end{array}$ \\
\hline $\begin{array}{l}\text { Charge and } \\
\text { discharge } \\
\text { rate }\end{array}$ & $\begin{array}{c}\text { Battery capacity is a certain value; the energy stored } \\
\text { and that released decreases with the current become } \\
\text { larger. Therefore, the battery capacity ratio must be } \\
\text { mentioned. }\end{array}$ \\
\hline $\begin{array}{c}\text { Self- } \\
\text { discharge }\end{array}$ & $\begin{array}{l}\text { Battery active material of anode and the positive } \\
\text { metal ions form the micro-cathode, the positive and } \\
\text { negative active material consumed when the battery } \\
\text { is stored for long-term, resulting in energy loss, this } \\
\text { phenomenon is the self-discharge }\end{array}$ \\
\hline
\end{tabular}

not match the expected output, then it goes to the BP stage. The error is transferred back in some form, from hidden layer to input layer and allocates the error to every unit of every layer, thus, the error signal of every layer is obtained and the error signal is taken as basis to amend the weights and threshold of every unit. The weight adjusting process, which contains signal forward propagation and error back propagation, the process goes continuously. The process of weight adjusting is the learning and training process of the network. The process continues until the output error reduces to an acceptable level or reaches the present training time [4]. Fig. 1 is the BP network model.

Network structure is dual-input and single-output, the number of nodes in the hidden layer is designed based on previous experience and experiment. In general, too many hidden nodes can lead to a long training time, while too few nodes can lead to poor fault-tolerance. As is shown in equation (2):

$$
n=\sqrt{n_{1}+n_{0}}+a
$$

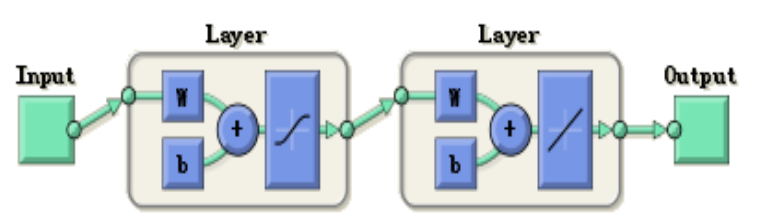

Figure 1. BP network model diagram
Where $n$ represents the hidden layer nodes, $n_{1}$ represents the input nodes, $n_{0}$ represents the output nodes; $a$ is a constant between 1 and 10. After experimental verification, the training time is appropriate and the faulttolerance is available when the hidden node is 6 .

Transfer function is the core of BP neural network, and is directly related to network performance. S-transfer function (tansig) is selected for the hidden layer transfer function, the output of this layer is limited between 0 and 1 , while the output layer transfer function is selected linear function, so any value can be achieved.

After the network structure is determined, an appropriate learning algorithm needs to be chosen for training the input and output of the network, which is to learn and amend the threshold of the network, to form the correspondent mapping from input to output. Traditional $\mathrm{BP}$ algorithm is a gradient descent learning algorithm, which is the same as the Widrow-Hoff rules, the value of the modified gradient descent along the reverse direction. This is prone to defects as follows: easy to fall into local minimum, more training times, low learning efficiency and slow convergence. For the problems mentioned above, there have been some improved algorithms, such as variant degree algorithm, Newton algorithm and momentum BP algorithm.

This paper selects LM (Levenberg-Marquardt) algorithm, which is proposed based on Newton algorithm, this method has local convergence of Newton algorithm and the global properties of gradient descent. In order to avoid the complex Hessian matrix computation, reduce the computational. Here, let $x^{(k)}$ denotes the iteration $\mathrm{k}$ vector of weights and thresholds, and the new vector $x^{(k+1)}$ can be obtained according to the following rules:

$$
x^{(k+1)}=x^{(k)}+\Delta x
$$

As for Newton algorithm the equation is:

$$
\Delta x=-\left[\nabla^{2} E(x)\right]^{-1} \nabla E(x)
$$

Where, $\nabla^{2} E(x)$ represents error indicator function, $E(x)$ is Hessian matrix, $\nabla E(x)$ means gradient. Hessian matrix can be approximated as:

$$
H=J^{T} J
$$

The gradient formula is

$$
g=J^{T} e
$$

Where, $J$ contains the network error function weights and threshold of the first derivative of the Jacobian matrix, $e$ is the error vector of the network, Jacobian matrix can be calculated by a standard former fed network technology, which is much simpler than Hessian matrix. As for LM method, the calculation rule is

$$
\Delta x=-\left[J^{T} J+\mu I\right]^{-1} J^{T} e
$$

Where, proportional coefficient $\mu$ is a constant bigger than $0, I$ is unit matrix.

Genetic algorithm is the operation of individual from population, usually consists of five basic elements: the parameter encoding, population initialization, fitness function, genetic manipulation and algorithm control mode. 
A. The parameter encoding-Genetic algorithm can not directly operate with the parameters of the problem space, it must take these parameters into space by the genetic structure of genes arranged in accordance with certain chromosome. This conversion is called encoding. Encoding requires the completeness, integrity and nonredundant of expression. The most commonly used encoding is the binary encoding, this method is consistent with the computer code, suitable for computer application, and each value of code string in the genetic expression is clear and easy to operate.

B. Population initialization-Prior to the genetic manipulation there must have one or more initial population. Initial population will directly affect the efficient of the algorithm. Commonly used method is selecting a certain number of individual randomly then selects the best individuals and inserts them into initialize the population, finally iterate until it reaches the preset scale.

C. Fitness function-In the process of biological evolution, fitness is the way to evaluate the ability of adapting to the environment, which is also the standard of survival of the fittest. Whether an individual fits the population is mainly based on fitness of individuals. In this way the optimal solution can be obtained. Therefore, fitness is an important factor of convergence rate and performance.

D. Genetic manipulation-Genetic manipulation is completely a simulation of the biological genetic approach. Use genetic operations of selection, crossover and mutation to complete the reorganization of gene sequences. Specific operation is as follows: first, select the individuals whose fitness are high from the population by using the method of roulette and then encode these individual following a certain probability of the genes, carry out the crossover operation, a new generation is obtained. The crossover operation is shown in Fig. 2.Finally, with a small probability mutation, the process avoids the permanent disappearance of certain genes caused by selection and crossover and it ensures the validity of the algorithm.

E. Algorithm control mode-Algorithm control including the set of population size, encoding length, selection probability, mutation probability and termination. Setting these parameters affect genetic algorithm performance inordinately. There is no formula or algorithm to determine these parameters, they can only be carried out through a lot of trial and error testing.

Although the BP neural network has a good generalization ability of nonlinear, the BP neural network use a gradient descent method, the value of the modified error function along the gradient BP. In practice, it prones to slow convergence, can not converge to the global minimum. Therefore, BP neural network is more suitable

$$
\text { Crossing Point }
$$

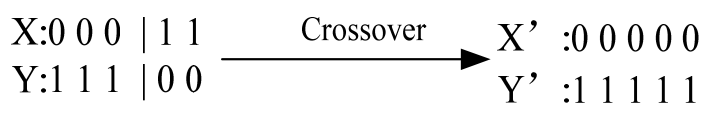

Figure. 2 Crossover Process for local search. We can use the global search ability of the genetic algorithm to optimize the initial. Fig. 3 shows the genetic neural network model structure.

To simplify the model, the inputs of the neural network are the battery charge current I and the voltage U, while the output layer is SOC. In Fig. 3, the initial weight threshold are provided by the genetic algorithm, BP neural network gets the actual output according to the input parameters, a comparison is made between the actual output and ideal output, the adjustment quantity of the weights and threshold value are obtained, the network parameters is adjusted, the cycle goes until the desired output or the terminating condition is met. The role of the genetic algorithm is to generate initial weights and threshold, this operation only run once.

Genetic algorithm is a random search method based on natural selection and the evolution of genetic mechanism in essence. It is based on the genetic evolution of biological theory and mathematical statistical theory. As for a population, the chromosome information is described through a string of binary code by encoding chromosomal gene which has entity characteristic, good population can be maintained according to the natural selection theory of Darwin, a better generation is produced. According to the actual problem, in the evolution of each generation, the individuals whose fitness are better are selected, and through the combination, crossover and mutation of genetics to generate new population. This generation will be able to simulate the natural evolution process of gradual adaptation to the environment, and the best individuals, namely the optimal solution, will come out by decoding [5] [6].

Neural network genetic algorithm optimization steps [7] are shown in Fig. 4.

A. Initializing the network and the population, give the training parameters, normalized each input so that the initial weights of the network is $[-1,1]$.

$B$. Calculating the fitness of each individual, because the population is the individual weights and threshold of the BP network, so make sure the selected fitness function is the inverse of error function for the network,

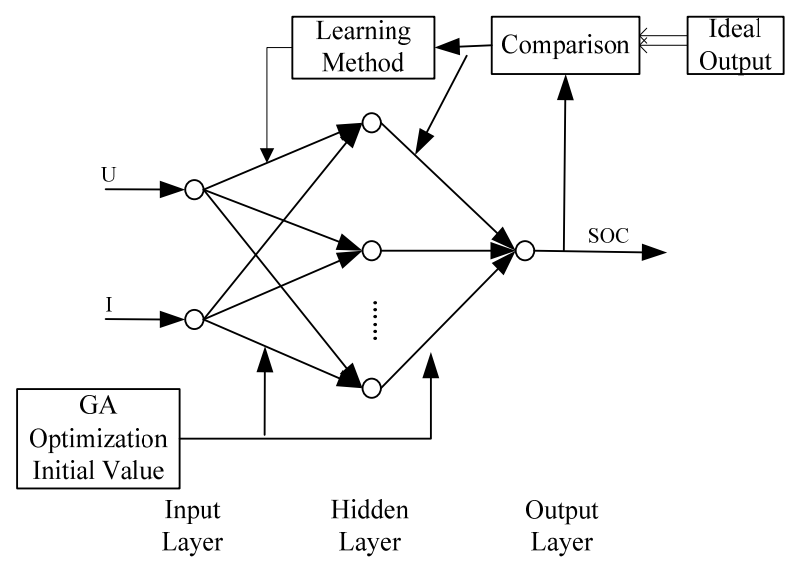

Figure 3. Genetic Neural Network Model Structure 


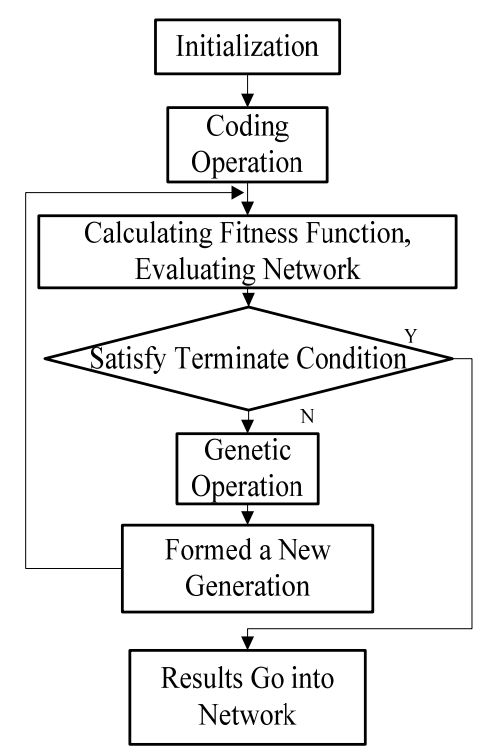

Figure 4. Genetic neural network optimization procedure

namely,

$$
\text { Fitness }(\text { sol })=\frac{1}{\sum_{i=1}^{\text {Popu }} E(\text { sol })}
$$

Where, sol represents each individual of the population, set bound is \pm 1 , Рорu represents the scale of population, its value is 50 . Because the structure of the BP neural network is 2-6-1, so the length of encode is $2 \times 6+6 \times 1+6+1=24$, by binary encoding.

$C$. Generic operation, the progeny population is obtained and the individuals are eliminated whose fitness is small by the operation selection, crossover and mutation. The crossover probability $P_{c}=0.7$, mutation probability $P_{m}=0.005$, the maximum evolution generation is 100 . Back to step $B$, until the fitness requirement or the maximum epoch is met.

$D$. Using the optimal initial value obtained by GA optimization to decode the value of initial weights and threshold obtained by BP neural network. Then BP neural network is trained, adjust the weights and threshold value to meet scheduled training accuracy and evaluate the performance of the network.

$E$. The final results obtained are the optimized estimation network.

\section{B. SOC Estimation Model Analysis}

A. The estimation model is clear, the method is simple and easy to be used in practical application.

$B$. Estimation model starts from the definition of SOC, effectively avoid the traditional problem of the need for specific model, using real-time acquisition of voltage and current as input and get corresponding relation through the network training, transfer them to microcontroller and look up the table.

$C$. Because of the prominent non-linear nature of the BP network, the learning method is flexible and is capable of massively parallel information processing.
$D$. Do the global search to get the initial weights and threshold of neural network by using the genetic algorithm. This can make neural network work better and can relieve and even eliminate local minima, slow convergence speed, etc.

$E$. Train the neural network by a large number of charge-discharge data, take advantage of the generalization ability of the network to improve the estimation accuracy. There is no requirement for types of the battery so this method can be applied to other types of battery.

F. In actual application, the weights and threshold values are stored in an array of MCU.

\section{SMUlation RESUlts ANALysis}

In order to verify the validity of the model, MATLAB software is used to simulate the experiments on the computer. First, modeling a framework according to the analysis of the previous section, and selecting the necessary parameters of $\mathrm{BP}$ network and genetic algorithm, then forming the code. The training data was taken under different charging rates from top to bottom are $4 \mathrm{C}, 2 \mathrm{C}, 1 \mathrm{C}, 0.5 \mathrm{C}$. The battery used in the experiment is composed of 6 single battery modules. Constant current power supply operating in constant pressure limiting state, whose constant current is 2C (16A), the maximum voltage is $9.5 \mathrm{~V}$. Network initial weights and threshold are obtained by genetic algorithm, optimization is given below. W1, B1 and $\mathrm{W} 2, \mathrm{~B} 2$ separately represent the weights and threshold from input layer to hidden layer and the hidden layer to output layer.

$$
\begin{array}{llllll}
\text { W1 }=0.3189 & 0.8059 & -0.6366 & -0.2589 & 0.6188 & -0.4155 \\
\text { W2 }=0.4501 & 0.4433 & -0.5089 & -0.1246 & 0.8905 & 0.4043 \\
\text { B1 }=-0.3452 & 0.2411 & -0.6178 & 0.2577 & -0.7750 & -0.3823 \\
\text { B2 }=0.3655 & & & & &
\end{array}
$$

Where the initial weights are between $0.1 \sim 0.9$ and network has a better convergence.

The non-optimized network training chart and the optimized chart are shown below in Fig. 5 and Fig. 6 .

At the same time using the mean square error as the BP and GA-BP evaluation of these two methods, and the training error and training epochs are shown in TABLE II.

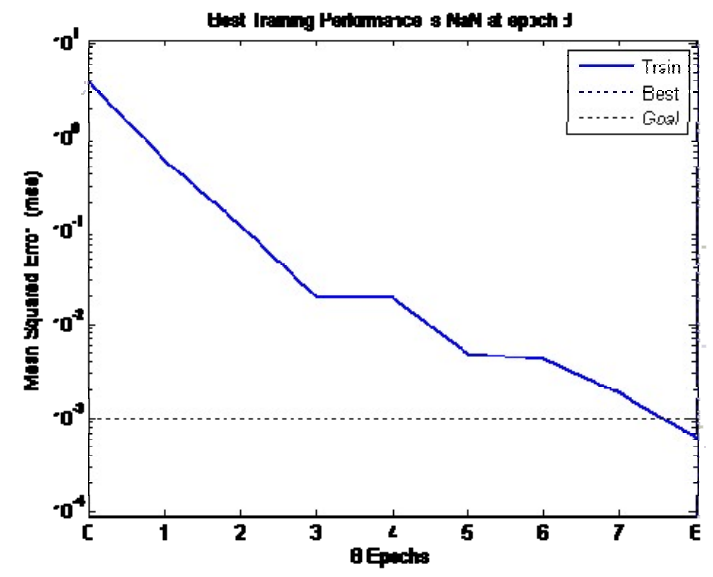

Figure 5. Non-optimized network training chart 


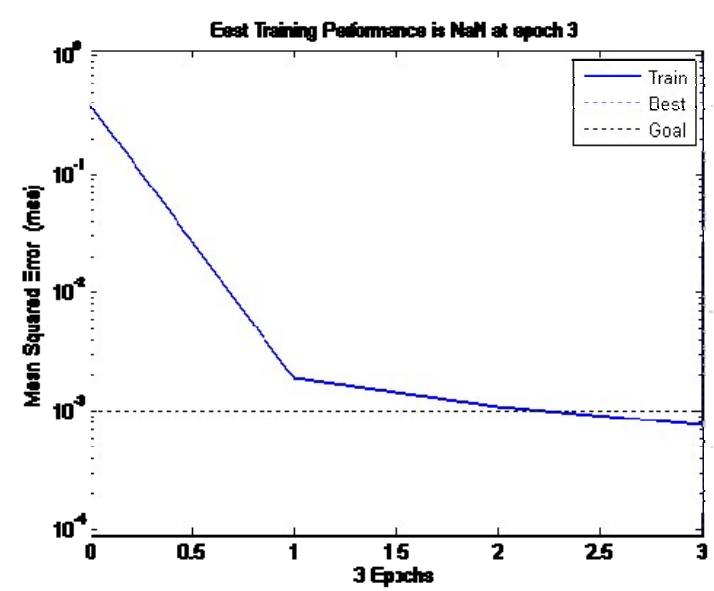

Figure 6. Optimized network training chart

As can be seen from TABLE II, comparing the training result of GA-BP method with that of $\mathrm{BP}$ network, the training epoch of the former was less than the latter, and the mean square error of GA-BP network is lower than traditional BP neural network, which proves the estimation of GA-BP method is better than BP method.

In Fig. 7 the solid line represents the GA-BP network trained curve, the data is selected SOC between $15 \%$ and $85 \%$. The dashed line is the real 2C of constant current charging SOC curve. It can be seen the prediction value is fluctuating up and down the real value, and the mean square error is less than $1 \%$, the simulation results well.

To verify the generalization ability of the network, choose the $0.5 \mathrm{C}(4 \mathrm{~A})$ and $3 \mathrm{C}(24 \mathrm{~A})$ of the constant current charging data as the input, the 45degree line in Fig. 8 and Fig. 9 represents the measured value and the real value perfectly matched. It can be seen, the predicted value has a good consistency with the experimental value. The mean square error is $1.603 \%$ and $2.0349 \%$, indicated that the network has good generalization ability.

\section{CONCLUSION}

This paper presents a GA-BP network method of battery SOC prediction based on the analysis of the common used estimation methods. A charging battery SOC prediction model of GA-BP network is built and using MATLAB 7 do simulation training and prediction experiment. The results show that after the training the power battery SOC can be predicted by collecting realtime voltage and current value. Power battery SOC prediction of GA-BP network method is not only theoretically feasible but with fast convergence and high accuracy, effectively reduces the shock of falling into

TABLE II

TRAINING ERROR AND EPOCH COMPARISONS BETWEEN BP AND GABP

\begin{tabular}{|c|c|c|}
\hline & BP & GA-BP \\
\hline$E_{M S E}$ & $0.9408 \%$ & $0.7577 \%$ \\
\hline Training Times & 8 & 3 \\
\hline
\end{tabular}

local minimum. Genetic algorithm combined with BP

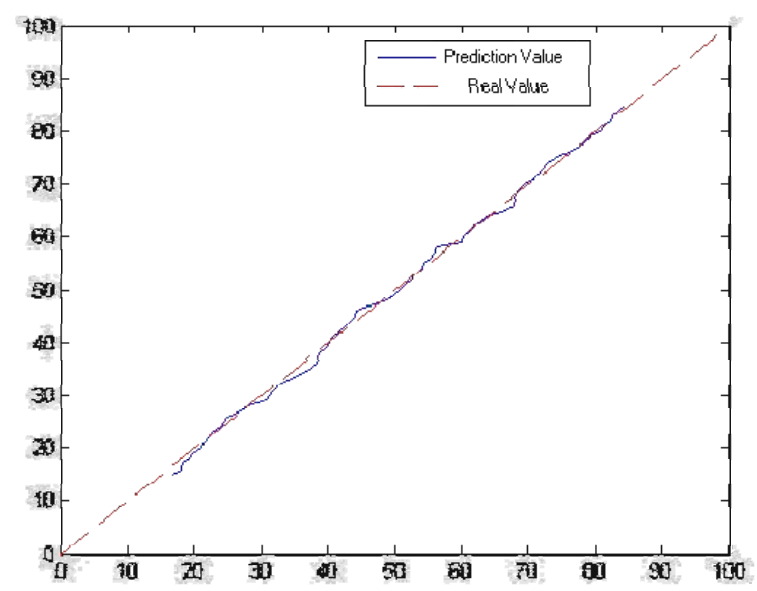

Figure 7. 2C constant current charging train

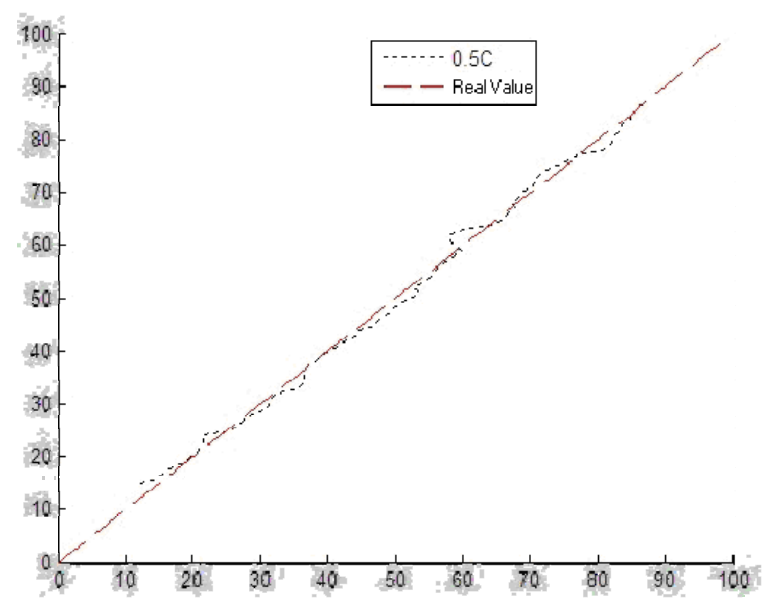

Figure 8. 0.5C SOC estimation curve

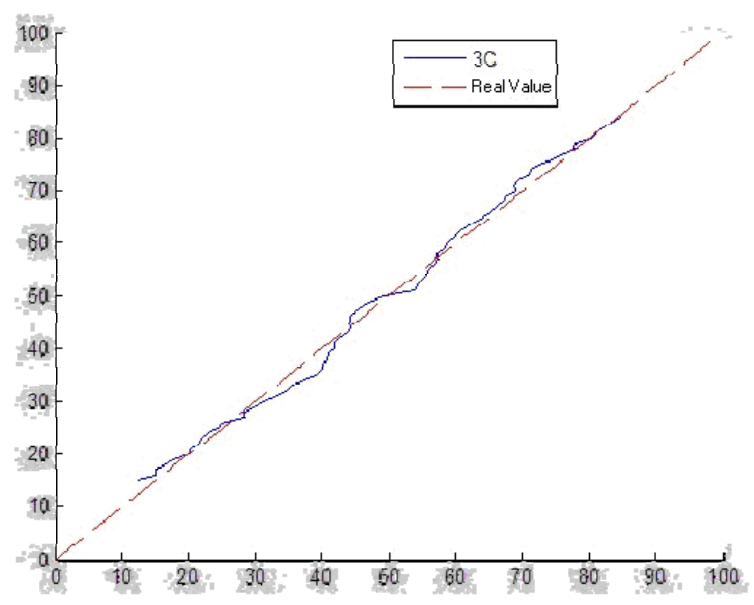

Figure 9. 3C SOC estimation curve

network effectively avoids the process of complex mathematical analysis, easy to practical application. A relatively accurate and efficient SOC prediction is provided for the power battery management system. 


\section{REFERENCES}

[1] José Luis Rojo-Álvarez,Manel Martínez-Ramón, Aníbal R. Figuriras-Vidal, et al. A robust support vector algorithm for nonparametric spectral analysis [J].IEEE, 2003,10 (11):320-323

[2] John Chiasson, Baskar Vairamohan. Estimating the State of Charge of a battery [J]. IEEE TRANSACTIONS ON CONTROL SYSTEMS TECHNOLOGY, 2005, 5, 13(3).

[3] Feisi technology product research center "Neural network theory and MATLAB7 implementation”, 2005, pp.99-108.

[4] Liqun Han, “Artificial neural network tutotial”,2006,pp.5878

[5] Yajun Wang, Xudong Wang, Yongqin Zhou, Yixin Yan, "GA-BP network based battery SOC prediction for quasi anti-damage power supply”, Electric Machines and Control, $6^{\text {th }}, 2010$, in press

[6] Xiaoping Wang, Liming Cao, "Genetic algorithms-Theory, Applications and Software”, 2002, pp.158-202.

[7] Jiansheng Wu, 'The Back-propagation Neural Network Meteorological Forecast Model Based on Genetic Algorithms”, 2004. in press.

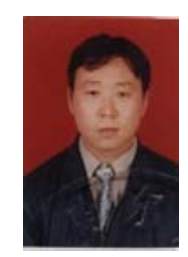

Yongqin Zhou was born in 1971, Henan province of China. He received M.S. degree in power electronics and electrical drives from Harbin University of Science and Technology in 2003.

He is currently a vice-professor of the Harbin University of Science and Technology and a member of Automobile Electronic Engineering Center. Some of the published articles are Research on Power Train Source Matching for Single-axle PHEV, 2008 IEEE Vehicle Power and Propulsion Conference, 2008, 9, and The Research and Realization for Passenger Car CAN Bus. Now he is doing the research on the Heilongjiang provincial science and technology key project, the development of hybrid car based on Hafeisaibao GB08A306 project.

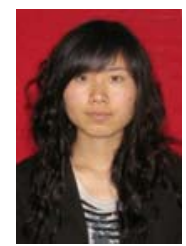

Chao Bai was born in 1985, Jilin province of China. She received B.S. degree in electrical engineering from Harbin University of Science and Technology, Heilongjiang, China, in 2008. She is a graduate student for a M.S. degree in the same school, under the guidance of Dr. Zhou.

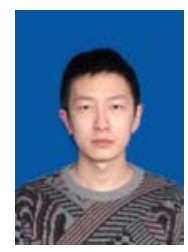

Jinlei Sun was born in 1985, Heilongjiang province of China. He received B.S. degree in electrical engineering from NorthEast Agriculture University in 2008, and M.S. degree in power electronics and electrical drives from Harbin University of Science and Technology, in 2011. His M.S. dissertation under the supervision of Dr. Zhou, was entitled "Battery SOC Estimation and System Implementation of Hybrid Electric Vehicle" 